\title{
Natural cycle versus hormone replacement therapy cycle in frozen-thawed embryo transfer
}

\author{
Ziya Kalem, MD, Müberra Namlı Kalem, MD, Batuhan Bakirarar, Statistician, Erkin Kent, MD, Timur Gurgan, MD.
}

\section{ABSTRACT}

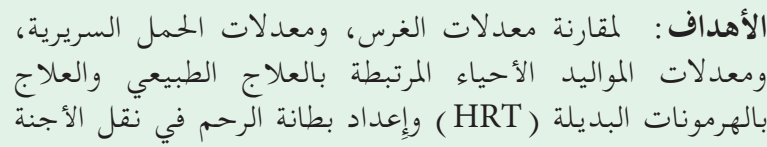

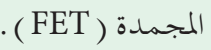

الطريقة: اشتملت النتائج على 108 دورات طبيعية و 224 دورة

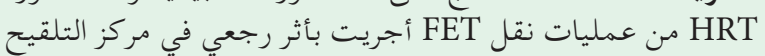

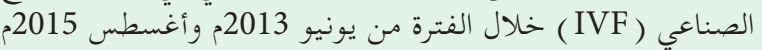

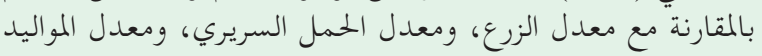
الأحياء.

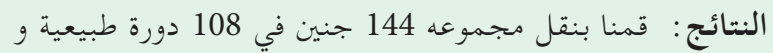

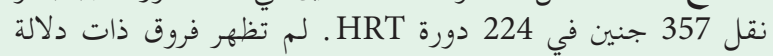

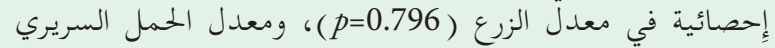

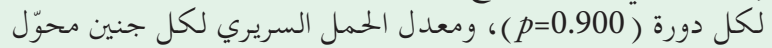

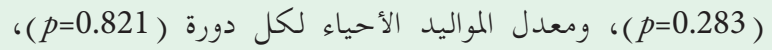

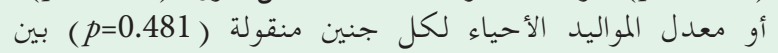
المجموعتين . - أو معدل

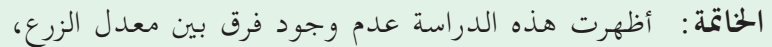

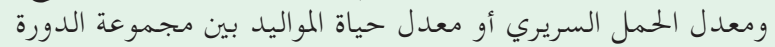

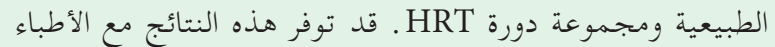

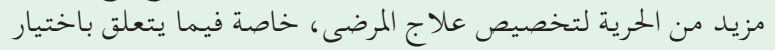

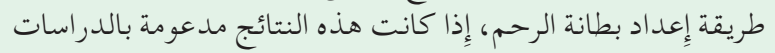

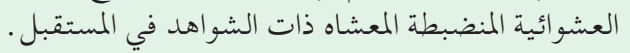

Objectives: To compare implantation rates, clinical pregnancy rates and live birth rates associated with natural and hormone replacement therapy (HRT) methods of endometrial preparation in frozen-thawed embryo transfer (FET) cycles.

Methods: The results of 108 natural cycles and 224 HRT cycles of FET transfers performed in a private in vitro fertilization (IVF) center between June 2013 and August 2015 were retrospectively compared with respect to implantation rate, clinical pregnancy rate, and live birth rate.
Results: A total of 144 embryos were transferred in 108 natural cycles and 357 embryos were transferred in 224 HRT cycles. No statistically significant differences were found in the implantation rate $(p=0.796)$, clinical pregnancy rate per cycle $(\mathrm{p}=0.900)$, clinical pregnancy rate per transferred embryo $(p=0.283)$, live birth rate per cycle $(p=0.821)$, or live birth rate per transferred embryo ( $p=0.481$ ) between the 2 groups.

Conclusion: This study showed no difference between the implantation rate, clinical pregnancy rate or live birth rate between the natural cycle group and HRT cycle group. These results may provide clinicians with more freedom to individualize patient treatment, particularly with respect to the selection of the endometrial preparation method, if these results are supported by large randomized controlled studies in the future.

\section{Saudi Med J 2018; Vol. 39 (11): 1102-1108} doi: 10.15537/smj.2018.11.23299

From the Department of IVF (Kalem, Kent, Gurgan), Gurgan Clinic, IVF and Women Health Center, from the Department of Obstetrics and Gynecology (Naml Kalem), Liv Hospital Ankara, from the Department of Biostatistic (Bakirarar), Ankara University, Ankara, and from the Department of Obstetrics and Gynecology (Gurgan), Bahcesehir University, Istanbul, Turkey.

Received 19th July 2018. Accepted 7th October 2018.

Address correspondence and reprint request to: Dr. Müberra N. Kalem, Department of Obstetrics and Gynecology, Liv Hospital Ankara, Ankara, Turkey.E-mail: muberranamli@hotmail.com

ORCID ID: orcid.org/0000-0002-2316-5495

Studies on cryopreservation of the human embryo $\checkmark$ and thawing and transferring it into the uterus began in the 1980s. ${ }^{1}$ In recent years, thaw cycles, during which the frozen-thawed embryos are transferred, have gained an increasingly important status in in vitro fertilization (IVF) protocols. ${ }^{2}$ There is no difference between the pregnancy and live birth results in fresh and thaw cycles. ${ }^{3}$ As the application of thaw cycles is being performed successfully more often, studies are being conducted to determine how to prepare the 
endometrium in which the embryo will be embedded. ${ }^{4,5}$ For a successful implantation, a receptive endometrium and synchronization between the endometrium and the embryo are essential. ${ }^{6}$ To date, several methods of endometrial preparation have been attempted. ${ }^{7}$ Spontaneous ovulation is monitored in natural cycles. In this technique, the time of embryo transfer is specified either by monitoring the spontaneous luteinizing hormone (LH) increase or by administering human chorionic gonadotropin (hCG) externally to induce ovulation. ${ }^{8,9}$ Furthermore, drug use is more frequent in hormone replacement therapy (HRT) cycles in which the endometrium is artificially prepared with estrogen and progesterone preparates. Therapy timing is also easier, and the number of follow-ups is lower. There are several studies on these methods and their modifications in the literature; ${ }^{10-13}$ however, there is no consensus on which method yields better results.

In the present study, we aimed to compare the pregnancy results of patients who underwent endometrial preparation with a natural cycle or an HRT cycle in freezing-thawing cycles.

Methods. This is a record-based study; data were examined from 536 IVF-applied cases with a frozenthawed embryo transfer (FET) cycle at a private IVF center between June 2013 and August 2015. From these cases, we selected healthy women who were not older than 40 years during the fresh cycle or freeze-thaw cycle, whose fresh and frozen-thawed cycles were performed in the same clinic and whose grade one embryos were used in both fresh and thaw cycles. Women older than 40 years, who had poor ovarian response to ovulation induction, ${ }^{14}$ from whom embryos were collected with the pooling system in a few fresh cycles, whose fertilization rate was lower than $50 \%$, who had systemic health problems or who were using antidiabetic, antihypertensive, or steroid-type medications were not included the study. A total of 332 individuals were included in the study, namely, 108 individuals in the natural cycle group and 224 in the HRT cycle group. The patients whose endometria were prepared with oral estrogen were not included in the HRT group, as they were few in number $(n=23)$; only patients in whom transdermal estrogen was used were included. Patients with irregular menstrual cycles were

Disclosure. Authors have no conflict of interests, and the work was not supported or funded by any drug company. included in the HRT group, but patients with regular menstrual cycles divided both HRT and natural cycle group according their clinical conditions. In the natural cycle group, only those patients whose menstrual cycles were regular were included.

Agonist and antagonist protocols were followed in patients' fresh cycles. In the agonist protocol, leuprolide acetate (Lucrin daily; Abbot, Cedex, France) was started at $10 \mathrm{U} /$ day in the mid-luteal phase of the previous cycle. The agonist dose was reduced to $5 \mathrm{U} /$ day and gonadotropin was added on the 3rd day of the menstrual cycle. On the third day of the antagonist cycle, recombinant gonadotropin (Puregon, MSD Organon, Oss, The Netherlands or Gonal-F, Merck-Serono, Geneva, Switzerland) was started, and on the sixth day of the gonadotropin treatment or when the dominant follicle was equal to or larger than $14 \mathrm{~mm}$, the gonadotropin-releasing hormone $(\mathrm{GnRH})$ antagonist cetrorelix $(0.25 \mathrm{mg}$; Cetrotide, Merck-Serono) was added. In both protocols, follicular development was followed up with ultrasonography (USG) (TVS; Voluson 730 expert; GE Medical Systems, Ltd., Hertfordshire, UK). When 3 dominant follicles that were $18 \mathrm{~mm}$ or larger were observed, 250 $\mu \mathrm{g}$ of recombinant(r)-hCG (Ovitrelle; Merck-Serono) or 10.000 IU urinary (u) hCG (Pregnyl; Organon, Skovlunde, Denmark) was administered, and oocyte retrieval was performed 36 hours following hCG injection. Intracytoplasmic sperm injection (ICSI) was performed on all the mature oocytes on the same day. The embryos were evaluated and transferred into the uterus between 2 and 5 days later. The embryos selected for freezing were frozen with vitrification between the third and fifth days. In this study, the vitrification and thawing procedure was performed using the Kitazato Vitrification Kit (Kitazato Co., Fujicity, Shizuoka, Japan) and open devices (Cryotech Reprolife Inc., Shinjuku, Tokyo, Japan) following the Kitazato protocol (300 $\mu$ l of equilibration solution for 12-15 minutes and $300 \mu \mathrm{l}$ of vitrification solution for $0.5 \pm 0.5$ minutes, followed by placement of the embryos with minimum volume). We also followed the Kitazato protocol for warming (thawing solution for $1 \mathrm{~min}$, dilution solution for $3 \mathrm{~min}$, warming solution for $5 \pm 1 \mathrm{~min}$ ). After the endometrium had been prepared in the thaw cycles, the embryos were thawed on the same day and transferred 3 to 6 hours after they were thawed.

For the preparation of patients in the natural cycle group, the follicles were followed up with USG at intervals of 3 to 4 days from the beginning of the cycle. When the dominant follicle was greater than $17 \mathrm{~mm}$, u-hCG 10000 U or r-hCG $500 \mu \mathrm{g}$ was administered. 
Progesterone was started 36 hours later, and natural progesterone (Crinone vaginal gel, Merck Serono, Switzerland) was used for 3 days in patients whose embryos were frozen on the third day and for 5 days in patients whose embryos were frozen on the fifth day, at $90 \mathrm{mg}$ twice a day. The embryos were transferred on the following day, and progesterone was continued in the women who became pregnant until the 10th to 12 th week of pregnancy.

The patients in the HRT cycle group were administered transdermal estrogen (Climara forte patch; Bayer, Istanbul, Turkey) at $100 \mu \mathrm{g}$ on the first day of the cycle, and it was changed every other day. The patients, whose doses were increased by 1 patch at intervals of 4 days, underwent USG on the 14th day of their cycle. The patients with an endometrial thickness of more than $8 \mathrm{~mm}$ started to receive vaginal natural progesterone (Crinone vaginal gel, Merck Serono) in the morning and at night on the following day (day 15). Following the 3-day progesterone (90 mg twice a day) use in patients whose embryos were frozen on the third day before transfer and 5-day progesterone use for those whose embryos were frozen on the fifth day before transfer, the embryos were thawed and transferred on the following day. After the transfer, estrogen and progesterone use was continued for 12 days until the beta-hCG test. Estrogen and progesterone use was continued in women who became pregnant until the 10th-12th week of pregnancy.

Statistical analysis. Statistical analysis was performed using the SPSS for Windows version 11.5 software (SPSS Inc., Chicago, IL, USA). The quantitative variables are expressed as the mean \pm standard deviation (SD), and categorical variables are expressed as the median (min-max) or as a number (percentage). To determine whether there was a statistically significant difference between the categories of qualitative and quantitative variables, student's t-test (normal distribution) or the Mann-Whitney U test (non-normal distribution) was used. The Chi-square test was used to analyze the relationship between the 2 categorical variables. To establish whether there was a statistically significant relationship between 2 quantitative variables, Pearson's correlation coefficient was used when both of the variables met the normal distribution assumptions, whereas Spearman's rank correlation coefficient was used when at least one of the variables did not meet the normal distribution assumptions. A $\mathrm{p}$ value of $<0.05$ was considered statistically significant.

Results. A total of 332 couples were included in the study. A natural cycle was applied to 108 of the women, while an HRT cycle was applied to the remaining 224 women. A total of $81.9 \%(n=264)$ of the couples had primary infertility, and $18 \% \quad(n=58)$ of them had secondary infertility. Regarding their pregnancy background, there were 45 chemical pregnancies, 89 miscarriages, 9 ectopic pregnancies, 23 live births vaginally, 32 cesarean sections $(\mathrm{C} / \mathrm{S})$, and 51 living children in total. In vitro fertilization indications were ovulatory dysfunction in $25.3 \%(n=84)$, male infertility in $34.9 \% \quad(\mathrm{n}=116)$, tubal factor in $11.1 \% \quad(\mathrm{n}=37)$, endometriosis in $9.6 \%(\mathrm{n}=32)$, and unexplained and other reasons in $18.9 \%(\mathrm{n}=63)$. Of the patients studied, $23.5 \%(n=78)$ were diagnosed with polycystic ovary syndrome (PCOS), while $76.5 \% \quad(\mathrm{n}=254)$ were not diagnosed with PCOS. Polycystic ovary syndrome was diagnosed in accordance with the Rotterdam ESHRE criteria. ${ }^{15}$

The mean endometrial thickness among the women with natural cycles on the hCG day was $11.6 \pm 1.8 \mathrm{~mm}$, and the median value was $12.0(6.0-18.0) \mathrm{mm}$. The mean endometrial thickness among the women with HRT cycles on the hCG day was $10.0 \pm 1.7 \mathrm{~mm}$, and the median value was $9.8(6.3-14.0) \mathrm{mm}$. Among the women with a HRT cycle, the mean number of patches was $18.2 \pm 3.8$ and median was $17.0(13.0-49.0)$. The mean HRT duration was $14.9 \pm 1.9$ and median was 15.0 (7.0-21.0) days. The mean endometrial thickness was $10.1 \pm 1.8$ and median and $10.0(5.0-14.0) \mathrm{mm}$, respectively. The mean and median oestradiol value on Day 15 was $632.9 \pm 194.3$ and 500.0 (72.0-1972.0) (pg/ $\mathrm{mL})$, respectively. The mean and median progesterone value was $0.6 \pm 0.5$ and $0.4(0.0-1.5) \mathrm{ng} / \mathrm{mL}$, respectively.

The women's age, age at the time of freezing, body mass index (BMI), day-3 oestradiol (E2), Day-3 progesterone $(\mathrm{P})$, infertility duration, number of previous fresh and thaw cycles, number of transferred embryos in the thaw cycle and the endometrial thickness on the transfer day are presented in Table 1.

In total, 539 embryos from 332 patients were thawed. Of these embryos, 375 were frozen in the cleavage stage (day 3) and 164 were frozen in the blastocyst stage (day 5). A total of 345 of 375 thawed embryos in the cleavage stage (92\%) and 156 of 164 thawed embryos in the blastocyst stage (95\%) were viable (survival rates of embryos in the cleavage state were $92 \%$ and blastocyst state were 95\%).

In total, 501 embryos were transferred, 144 in the natural cycle group (108 cycles) and 357 in the HRT group (224 cycles). In the natural cycle group, 101 $(70.1 \%)$ of the embryos were in the cleavage stage, while 
$43(29.8 \%)$ were in the embryo blastocyst stage. In the HRT cycle group, 244 (68.4\%) of the embryos were transferred in the cleavage stage, while 113 (31.6\%) were transferred in the blastocyst embryo stage.

No statistically significant difference was found between cleavage embryo implantation rate $(p=0.686)$ or blastocyst embryo implantation rate $(p=0.403)$ in all patients. Additionally, there was no statistically significant difference between cleavage embryo live birth rate $(p=0.503)$ and blastocyst embryo live birth rate $(p=0.403)$ in this study.

Comparisons of natural cycle and HRT cycle outcomes are presented in Table 2 .

In the fresh cycles of the participants, rec-FSH $(75.6 \%)$ or urinary FSH (24.4\%) was used for ovulation induction, and recombinant hCG (52.7\%), urinary hCG $(43.0 \%)$ or agonist trigger $(4.3 \%)$ was used to trigger ovulation. Early ovarian hyperstimulation syndrome (OHSS) developed in $18(5.5 \%)$ of the women, and late OHSS developed in $3(0.9 \%)$ of the women. At the end of the fresh cycles, $178(53.6 \%)$ patients underwent embryo transfer. The clinical pregnancy rate was $34.2 \%$ $(n=61)$, and the live birth rate was $25.2 \%(n=45)$ in the fresh cycles. Other parameters of the fresh cycles of the individuals who participated in the study are presented in Table 3.

There was no statistically significant correlation between the live birth rate after the fresh cycle and the live birth rate after the thaw cycle in the same patient $(p=0.852, \mathrm{r}=-0.017)$.

Table 1 - Baseline characteristics of the natural cycle and HRT cycle groups

\begin{tabular}{|c|c|c|c|c|c|}
\hline \multirow[t]{3}{*}{ Variables } & \multicolumn{5}{|c|}{ Cycle groups } \\
\hline & \multicolumn{2}{|c|}{ Natural cycle $(n=108)$} & \multicolumn{2}{|c|}{ HRT cycle $(n=224)$} & \multirow[b]{2}{*}{$P$-value } \\
\hline & Mean \pm SD & $\begin{array}{c}\text { Median } \\
\text { (Min - Max) }\end{array}$ & Mean \pm SD & $\begin{array}{c}\text { Median } \\
\text { (Min - Max) }\end{array}$ & \\
\hline Age at thawing (years) & $33.30 \pm 5.37$ & $\begin{array}{c}33.00 \\
(21.00-40.00)\end{array}$ & $32.60 \pm 5.69$ & $\begin{array}{c}32.00 \\
(18.00-40.00)\end{array}$ & 0.121 \\
\hline Age at freezing (years) & $30.94 \pm 5.21$ & $\begin{array}{c}30.70 \\
(19.00-40.00)\end{array}$ & $30.22 \pm 6.13$ & $\begin{array}{c}30.10 \\
(19.00-40.00)\end{array}$ & 0.231 \\
\hline BMI $\left(\mathrm{kg} / \mathrm{m}^{2}\right)$ & $24.91 \pm 4.02$ & $\begin{array}{c}24.54 \\
(18.20-36.00)\end{array}$ & $25.27 \pm 4.50$ & $\begin{array}{c}24.61 \\
(16.52-46.65)\end{array}$ & 0.648 \\
\hline Day-3 $E_{2}(\mathrm{pg} / \mathrm{ml})$ & $38.33 \pm 25.58$ & $\begin{array}{c}60.00 \\
(19.00-66.00)\end{array}$ & $38.96 \pm 18.99$ & $\begin{array}{c}42.00 \\
(2.00-87.00)\end{array}$ & 0.330 \\
\hline Day-3 progesterone $(\mathrm{ng} / \mathrm{ml})$ & $0.26 \pm 0.07$ & $\begin{array}{c}0.26 \\
(0.21-0.31)\end{array}$ & $0.48 \pm 0.33$ & $\begin{array}{c}0.40 \\
(0.08-1.69)\end{array}$ & $<0.001$ \\
\hline Duration of infertility (years) & $7.19 \pm 4.58$ & $\begin{array}{c}6.00 \\
(1.00-20.00)\end{array}$ & $7.33 \pm 4.51$ & $\begin{array}{c}6.50 \\
(1.00-22.00)\end{array}$ & 0.721 \\
\hline Previous fresh cycle number & $2.92 \pm 2.54$ & $\begin{array}{c}2.00 \\
(1.00-15.00)\end{array}$ & $2.44 \pm 2.41$ & $\begin{array}{c}2.00 \\
(0.00-18.00)\end{array}$ & 0.101 \\
\hline Previous thaw cycle number & $1.33 \pm 0.91$ & $\begin{array}{c}1.00 \\
(0.00-4.00)\end{array}$ & $1.12 \pm 0.83$ & $\begin{array}{c}1.00 \\
(0.00-3.00)\end{array}$ & 0.318 \\
\hline Number of transferred embryos & $1.53 \pm 0.52$ & $\begin{array}{c}2.00 \\
(1.00-3.00)\end{array}$ & $1.60 \pm 0.53$ & $\begin{array}{c}2.00 \\
(1.00-4.00)\end{array}$ & 0.187 \\
\hline $\begin{array}{l}\text { Endometrial thickness on embryo transfer day } \\
(\mathrm{mm})\end{array}$ & $11.56 \pm 2.61$ & $\begin{array}{c}11.75 \\
(6.40-15.80) \\
\end{array}$ & $10.74 \pm 2.35$ & $\begin{array}{c}10.90 \\
(6.11-19.20) \\
\end{array}$ & 0.117 \\
\hline
\end{tabular}

Table 2 - Comparison of natural and hormonal replacement treatment (HRT) cycle outcomes.

\begin{tabular}{lrrr}
\hline Results & \multicolumn{2}{c}{ Natural cycle } & \multicolumn{2}{c}{ HRT cycle } & $P$-value \\
& $\mathbf{n}(\%)$ & n $(\%)$ & \\
\hline Positive pregnancy test & $47 / 108(43.5)$ & $94 / 224(41.9)$ & 0.411 \\
Chemical pregnancy & $6 / 108(5.6)$ & $15 / 224(6.69)$ & 0.689 \\
Abortion & $4 / 108(3.7)$ & $9 / 224(4.0)$ & 1.000 \\
Twin pregnancy & $6 / 108(5.6)$ & $10 / 224(4.5)$ & 0.664 \\
Implantation rate (IR) & $45 / 108(41.7)$ & $90 / 224(40.2)$ & 0.796 \\
Clinical pregnancy rate (per cycle) & $34 / 108(31.5)$ & $69 / 224(30.8)$ & 0.900 \\
Clinical pregnancy rate (per embryo) & $34 / 144(23.6)$ & $69 / 357(19.3)$ & 0.283 \\
Live birth rate (per cycle) & $31 / 108(28.7)$ & $67 / 224(29.9)$ & 0.821 \\
Live birth rate (per embryo) & $31 / 144(21.5)$ & $67 / 357(18.8)$ & 0.481 \\
\hline
\end{tabular}


Table 3 - Fresh cycle parameters of 322 patients included in the study.

\begin{tabular}{lcc}
\hline Fresh cycle $(\mathbf{n}=322)$ & Mean \pm SD & Median $($ Min - Max $)$ \\
\hline AFC & $13.22 \pm 6.40$ & $14.00(1.00-20.00)$ \\
D3E2 $(\mathrm{ng} / \mathrm{ml})$ & $31.12 \pm 17.63$ & $28.00(1.00-97.00)$ \\
D3P $(\mathrm{pg} / \mathrm{ml})$ & $0.44 \pm 0.32$ & $0.40(0.00-1.80)$ \\
Number of expected oocytes & $10.34 \pm 5.72$ & $10.00(1.00-28.00)$ \\
Number of retrieved oocytes & $7.53 \pm 4.81$ & $7.00(1.00-35.00)$ \\
Number of mature oocytes & $6.23 \pm 4.10$ & $6.00(1.00-35.00)$ \\
Number of fertilized oocytes (2PN) & $5.80 \pm 3.92$ & $6.00(1.00-33.00)$ \\
Fertilization rate $(\%)$ & $94.03 \pm 12.72$ & $100.00(40.00-100.00)$ \\
Number of good-quality embryos & $3.52 \pm 2.41$ & $3.00(1.00-16.00)$ \\
Number of transferred embryos & $1.43 \pm 0.64$ & $1.00(0.00-4.00)$ \\
Number of frozen embryos & $3.71 \pm 2.84$ & $3.00(1.00-17.00)$ \\
Number of days frozen & $3.24 \pm 0.80$ & $3.00(2.00-6.00)$ \\
Number of frozen embryo cells & $10.61 \pm 7.32$ & $8.00(2.00-30.00)$ \\
GRADE-1 embryos & $2.92 \pm 2.14$ & $2.00(0.00-16.00)$ \\
GRADE-2 embryos & $2.23 \pm 1.40$ & $2.00(1.00-7.00)$ \\
GRADE-3 embryos & $2.21 \pm 1.81$ & $1.00(1.00-7.00)$ \\
\hline
\end{tabular}

Discussion In this study, in which the natural cycles and HRT cycles used in the preparation of the endometrium in thaw cycles were compared, the implantation rate, clinical pregnancy rate and live birth rate were similar between the 2 groups. No correlation was found between the live birth rate in the fresh cycle and the live birth rate in the thaw cycle.

In the literature, different results have been published in studies comparing natural and HRT cycles. Morozov et $\mathrm{al}^{16}$ found that the natural cycle group had a higher pregnancy rate, endometrial thickness, and estradiol level and that high estradiol in the HRT cycle group impaired endometrial receptivity during the implantation window and lowered the pregnancy rate. Xiao et $\mathrm{al}^{17}$ found fewer biochemical pregnancies and higher implantation and ongoing pregnancy rates in the natural cycle group than in the HRT cycle group. Similarly, Levron et $\mathrm{a}^{18}$ showed, in a retrospective study in which 1235 FET cycles were evaluated over a period of 12 years, that endometrial thickness, implantation rate and clinical pregnancy rate were higher in the natural-cycle FET. In a study performed by Givens et $\mathrm{al},{ }^{19}$ the pregnancy rate was higher in the HRT cycle group than the natural cycle group. In the same study, the miscarriage rate was higher in the HRT group, and there was no difference between the live birth rates. In a retrospective study conducted by Zheng et al, ${ }^{20}$ in which 5414 patients were examined, implantation and clinical pregnancy rates were increased in the HRT cycles. The same study reported that ovulation in HRT cycles had a negative impact on pregnancy, and in the FET cycles, ovulation could be followed up well and the cycle could be cancelled. Gelbaya et $\mathrm{al}^{8}$ found that implantation, pregnancy and live birth rates were similar in the natural and hormone-controlled groups of patients whose menstrual cycles were regular. In a randomized controlled study only involving patients who were ovulatory and had regular menstrual cycles, FET outcomes were similar between the natural and HRT cycle groups. ${ }^{21}$ Several studies reported that a thick endometrium has a positive impact on pregnancy rate. ${ }^{22-24}$ Some authors anticipated that the endometrial thickness would increase as estradiol increased in HRT cycles; ${ }^{25-27}$ however, no significant changes were observed in endometrial thicknesses. ${ }^{27,28}$ Meanwhile, discussions on the detrimental effect of supra-physiological estradiol levels on endometrial quality continue. If estradiol levels were actually higher in HRT cycles and this negatively affected implantation in the endometrium, it could be expected that pregnancy rates would be lower in HRT cycles. In this study, no significant difference was found between the 2 groups in terms of endometrial thickness or pregnancy rate.

Patients who were using transdermal estrogen in HRT cycles were selected for this study. Because a limited number of patients were using oral tablets, it was anticipated that this group would be statistically inadequate, so the patients in this group were excluded from the study. Transdermal estrogen was previously preferred at our clinic due to its ease of administration, as well as the notion that it is more physiological. Part of the estradiol valerate administered orally is transformed into estrone in the intestinal system. ${ }^{29}$ Estradiol and estrone are carried to the liver through the portal system, where they are transformed into estriol. During this transition, estrogen's activity in the circulation decreases 
by $30 \% \cdot{ }^{30}$ When estrogen is administered transdermally, no metabolism occurs in the liver; therefore, estradiol concentrations are greater than estrone concentrations. An estradiol/estrone ratio of approximately 1 is found, which is more compatible with human physiology. ${ }^{31}$ When estrogen is administered orally, this ratio is 0.2 , namely, much lower than physiological values. ${ }^{32}$ There is no difference between oral and transdermal estradiol use in thaw cycles in terms of serum estradiol level and endometrial thickness. ${ }^{4,33}$ In 2 studies investigating the effect of luteal support in natural cycles, it was shown that when luteal support was given, it did not affect the outcome in terms of clinical pregnancy; however, a significant difference was found in ongoing pregnancy in favor of luteal support. ${ }^{28,34}$ In this study, all patients were administered progesterone for luteal support, and it was continued when pregnancy was in place until the 10 th to 12 th week.

Study limitation. The most important limitation of this study is its retrospective design. Thus, the numbers of participants in the 2 groups are unequal. Small sample size and low power also important limitations of the study. In addition, estradiol levels were examined on day 15 in HRT cycles but not in the spontaneous cycles, which is why no comparison was made between the groups in terms of estradiol. No significant difference was found between the groups in terms of their demographics, histories or cycle parameters, although the study was retrospective. These similarities make the study more valuable, as the groups were homogenous. Most of the thaw cycle studies in the literature have been performed with patients whose cycles are regular. ${ }^{10,16,18,20}$ This study included patients with regular cycles only in the natural cycle group, while there were patients with regular and irregular cycles in the HRT group. Nevertheless, the similar pregnancy outcomes showed that cycle order is not important in the HRT cycle. However, the creation of a PCOS group or irregular cycle group as a separate subgroup would have added more value to the study.

In conclusion, this study compared the success of endometrial preparation methods in freeze-thaw cycles. Our results showed no difference between the implantation rate, clinical pregnancy rate or live birth rate between the natural cycle group and HRT cycle group. The similar results of the 2 groups may provide clinicians with more freedom to individualize patient treatment. As the number of FET transfer cycles increases in clinical practice, further randomized controlled studies with higher numbers of cycles would help guide clinicians in the selection of endometrial preparation techniques.

\section{References}

1. Trounson A, Mohr L. Human pregnancy following cryopreservation, thawing and transfer of an eight-cell embryo. Nature 1983; 305: 707-709.

2. Ferraretti AP, Goossens V, Kupka M, Bhattacharya S, de Mouzon J, Castilla JA, Partnerschaft S. Assisted reproductive technology in Europe, 2009: results generated from European registers by ESHRE. Human Reprod 2013; 28: 2318-2331.

3. Crawford S, Boulet SL, Kawwass JF, Jamieson DJ, Kissin DM. Cryopreserved oocyte versus fresh oocyte assisted reproductive technology cycles, United States, 2013. Fertil Steril 2017; 107: 110-118.

4. Ghobara T, Vanderkerchove P. Cycle regimens for frozen thawed embryo transfer. Cochrane Database Syst Rev 2008; (1): CD003414.

5. Groenewoud ER, Cantineau AE, Kollen BJ, Macklon NS, Cohlen BJ. What is the optimal means of preparing the endometrium in frozen-thawed embryo transfer cycles? A systematic review and meta-analysis. Hum Reprod Update 2013; 19: 458-470.

6. Shapiro BS, Daneshmand ST, Garner FC, Aguirre M, Hudson C, Thomas S. Evidence of impaired endometrial receptivity after ovarian stimulation for in vitro fertilization: a prospective randomized trial comparing fresh and frozen-thawed embryo transfer in normal responders. Fertil Steril 2011; 96: 344-348.

7. Kalem Z, Kalem MN, Gürgan T. Methods for endometrial preparation in frozen-thawed embryo transfer cycles. $J$ Turk Ger Gynecol Assoc 2016; 17: 168-172.

8. Gelbaya TA, Nardo LG, Hunter HR, Fitzgerald CT, Horne G, Pease EE, et al. Cryopreserved-thawed embryo transfer in natural or down-regulated hormonally controlled cycles: a retrospective study. Fertil Steril 2006; 85: 603-609.

9. Pelinck MJ, Vogel NE, Arts EG, Simons AH, Heineman MJ, Hoek A. Cumulative pregnancy rates after a maximum of nine cycles of modified natural cycle IVF and analysis of patient drop-out: a cohort study. Hum Reprod 2007; 22: 2463-2470.

10. Groenewoud ER, Cohlen BJ, Al-Oraiby A, Brinkhuis EA, Broekmans FJ, de Bruin JP, et al. A randomized controlled, non-inferiority trial of modified natural versus artificial cycle for cryo-thawed embryo transfer. Hum Reprod 2016; 31: 1483-1492.

11. Orvieto R, Feldman N, Lantsberg D, Manela D, Zilberberg E, Haas J. Natural cycle frozen-thawed embryo transfer-can we improve cycle outcome? J Assist Reprod Genet 2016; 33 : 611-615.

12. Arefi S, Hoseini A, Farifteh F, Zeraati H. Modified natural cycle frozen-thawed embryo transfer in patients with repeated implantation failure: An observational study. Int J Reprod Biomed (Yazd) 2016; 14: 465-470.

13. Jouan C, Emonard V, Ruggeri P, Debelle L, Hincourt N, Lorquet $S$, et al. Pregnancy outcome following frozen embryo transfer after artificial cycle or treatment by clomiphene citrate. Gynecol Endocrinol 2016; 32: 807-810.

14. Ferraretti AP, La Marca A, Fauser BC, Tarlatzis B, Nargund G, Gianaroli L, et al. ESHRE consensus on the definition of 'poor response'to ovarian stimulation for in vitro fertilization: the Bologna criteria. Hum Reprod 2011; 26: 1616-1624. 
15. Rotterdam ESHRE/ASRM-Sponsored PCOS Consensus Workshop Group. Revised 2003 consensus on diagnostic criteria and long-term health risks related to polycystic ovary syndrome. Fertil Steril 2004; 81: 19-25.

16. Morozov V, Ruman J, Kenigsberg D, Moodie G, Brenner S. Natural cycle cryo-thaw transfer may improve pregnancy outcome. J Assist Reprod Genet 2007; 24: 119-123.

17. Xiao Z, Zhou X, Xu W, Yang J, Xie Q. Natural cycle is superior to hormone replacement therapy cycle for vitrificated-preserved frozen-thawed embryo transfer. Syst Biol Reprod Med 2012; 58: $107-112$.

18. Levron J, Yerushalmi GM, Brengauz M, Gat I, Katorza E. Comparison between two protocols for thawed embryo transfer: natural cycle versus exogenous hormone replacement. Gynecol Endocrinol 2014; 30: 494-497.

19. Givens CR, Markun LC, Ryan IP, Chenette PE, Herbert CM, Schriock ED. Outcomes of natural cycles versus programmed cycles for 1677 frozen-thawed embryo transfers. Reprod Biomed Online 2009; 19: 380-384.

20. Zheng Y, Li Z, Xiong M, Luo T, Dong X, Huang B, et al. Hormonal replacement treatment improves clinical pregnancy in frozen-thawed embryos transfer cycles: a retrospective cohort study. Am J Transl Res 2013; 6: 85-90.

21. Mounce G, McVeigh E, Turner K, Child TJ. Randomized, controlled pilot trial of natural versus hormone replacement therapy cycles in frozen embryo replacement in vitro fertilization. Fertil Steril 2015; 104: 915.e1-920.e1.

22. Richter KS, Bugge KR, Bromer JG, Levy MJ. Relationship between endometrial thickness and embryo implantation, based on 1,294 cycles of in vitro fertilization with transfer of two blastocyst-stage embryos. Fertil Steril 2007; 87: 53-59.

23. El-Toukhy T, Coomarasamy A, Khairy M, Sunkara K, Seed P, Khalaf Y, et al. The relationship between endometrial thickness and outcome of medicated frozen embryo replacement cycles. Fertil Steril 2008; 89: 832-839.

24. Kumbak B, Erden HF, Tosun S, Akbas H, Ulug U, Bahçeci M. Outcome of assisted reproduction treatment in patients with endometrial thickness less than $7 \mathrm{~mm}$. Reprod Biomed Online 2009; 18: 79-84.
25. de Ziegler D, Cornel C, Bergeron C, Hazout A, Bouchard P, Frydman R. Controlled preparation of the endometrium with exogenous estradiol and progesterone in women having functioning ovaries. Fertil Steril 1991; 56: 851-855.

26. Griesinger G, Weig M, Schroer A, Diedrich K, Kolibianakis EM. Mid-cycle serum levels of endogenous LH are not associated with the likelihood of pregnancy in artificial frozenthawed embryo transfer cycles without pituitary suppression. Human Reprod 2007; 22: 2589-2593.

27. Hancke K, More S, Kreienberg R, Weiss JM.Patients undergoing frozen-thawed embryo transfer have similar live birth rates in spontaneous and artificial cycles. J Assist Reprod Genet 2012; 29: 403-407.

28. Tomás C, Alsbjerg B, Martikainen H, Humaidan P. Pregnancy loss after frozen-embryo transfer-a comparison of three protocols. Fertil Steril 2012; 98: 1165-1169.

29. van Keep PA, Utian WH, Vermeulen A. Potency and hepato-cellular effects of oestrogens after oral, percutaneous, and subcutaneous administration. In: van Keep PA, Utian WH, Vermeulen A, editors. The Controversial Climacteric. Netherlands: Springer, Dordrecht; 1982 p. 103-125.

30. Shufelt CL, Merz CN, Prentice RL, Pettinger MB, Rossouw JE, Aroda VR, et al. Hormone therapy dose, formulation, route of delivery, and risk of cardiovascular events in women: findings from the WHI observational study. Menopause 2014; 21: 260-266.

31. Chetkowski RJ, Meldrum DR, Steingold KA, Randle D, Lu JK, Eggena P, et al. Biologic effects of transdermal estradiol. $N$ Engl J Med 1986; 314: 1615-1620.

32. Powers MS, Schenkel L, Darley PE, Good WR, Balestra JC, Place VA. Am J Obstet Gynecol 1985; 152: 1099-106.

33. Davar R, Janati S, Mohseni F, Khabazkhoob M, Asgari S. A comparison of the effects of transdermal estradiol and estradiol valerate on endometrial receptivity in frozen-thawed embryo transfer cycles: a randomized clinical trial. J Reprod Infertil 2016; 17: 97-103.

34. Weissman A, Horowitz E, Ravhon A, Steinfeld Z, Mutzafi R, Golan A, Levran D. Spontaneous ovulation versus HCG triggering for timing natural-cycle frozen-thawed embryo transfer: a randomized study. Reprod Biomed Online 2011; 23: 484-489. 